\title{
Computer Aided Simulation of Hydroforming Process
}

\author{
S.B.Nandeppagoudar ${ }^{1,2}$, S.S.Ghorpade ${ }^{1}$, S.H.Gawande ${ }^{1}$ \\ ${ }^{l}$ (Department of Mechanical Engineering, M.E.S.College of Engineering, S.P.Pune University,India) \\ ${ }^{2}$ (Naveen Hydro-controls, Bhosari MIDC, Pune, India)
}

\begin{abstract}
Modern manufacturing technology paternity is always finding some or the other alternative methods of manufacturing processes in hydroforming. Stream Hydroforming is one of the super fine metals forming process. From last four to five decades many applications have come in existence. Recently hydroforming has been applied in automotive components however it has many challenges such as metallurgical aspects of materials, tribological aspects, die design, non-availability of high pressure components for equipment manufacturing and lack of literature and references. In this study, Finite elementanalysis (FEA) for simple shape was performed and metal forming behavior during hydroforming was observed. In this study the influence of the axial movement and pressure applied on the confined fluid (water) in the tube is recorded and it is correlated as under- "The axial movement of tube is directly related with change in internal volume of the tube". Here we found that the potential of this hydroforming method in the sheet metal manufacturing industries is very large.
\end{abstract}

Keywords: Formability,High pressure, Hydroforming, Pre-forming, Sheet Hydroforming, Simulation,.

\section{INTRODUCTION}

Tube hydroforming (THF) is a one of the recently used unconventional metal forming process in automotive and aerospace industry to produce hollow shapes with complex geometriesas shown in Fig.1, and Fig.2 by using axial feed and internal pressure. Tube Hydroforming is now taking lead in modern manufacturing process which is replacing many subassembly processes and therefore this process is becoming more popular and most efficient. In recent years more researches havebeen done on Tube hydroforming by using FEA software such as solidworkssimulation, LS-DYNA, ABAQUS, PAM-STAMP, AUTO-FORM, DE-FORM. These are used to study and analyse the various processes parameters like axial compressive force and internal pressure on the fluid, tribological effect, thinning of tube material. Most failure modes in THF are wrinkling, buckling, bursting. These types of failures are caused by either excessive internal pressure on the liquid or excessive axial feed from both ends during the forming process. The procedure of free bulge test is simple; a metal tubular specimen is held in dies withinternal pressure and expands, undergoing plastic deformationuntil bursting occurs. During the process the tubes locked onboth ends and applied the axial feed using hydraulic actuators along with internalpressure which will reduce the thinning of tube material as shown in Fig.3. This paper aims to analyze the hydroformingprocess by using SolidWorkSimulation software and compare thesimulation results with experimental results.

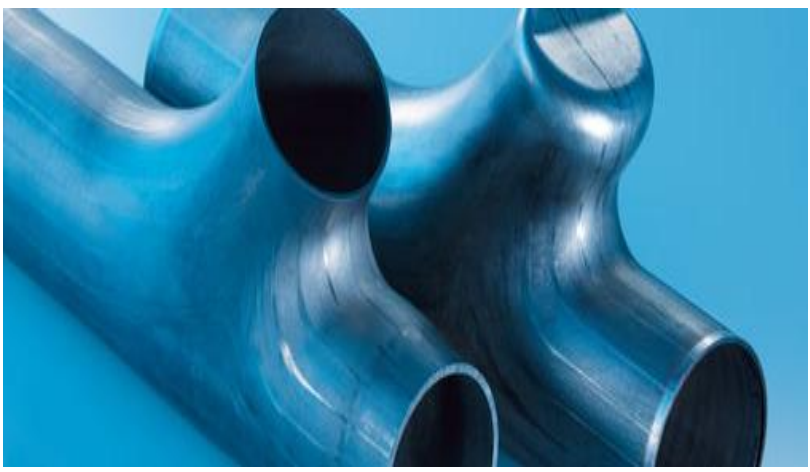

Fig. 1 Y-type hydro-formed parts

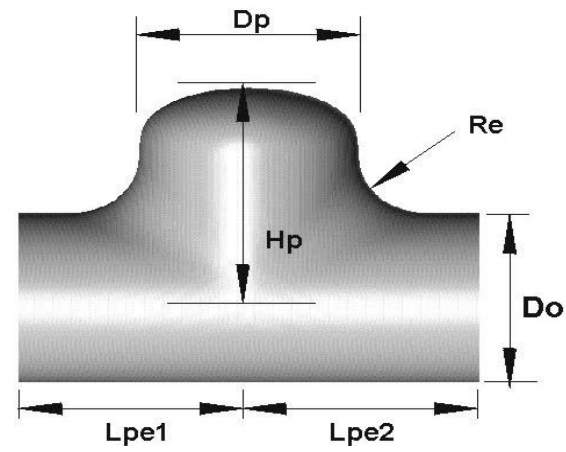

Fig. 2 T- type hydro-formed parts 
This process THF provides a number of advantages in comparison with conventional manufacturing processes like stamping, welding and riveting etc. are as under:

- Part consolidation resulting in weight reduction of the component.

- Weight reduction through more efficient section design by optimum thickness.

- Reduced tooling cost, dimensional stability of components and optimum usage of raw material and resources.

- Improved structural strength and stiffness.

- Reduce number of subassembly parts and auxiliary operations.

- Due to which main assembly will become simpler and efficient.

- Improved productivity and hence overall reduction in manufacturing cost.

- Improved dimensional stability due to uniform load on entire inner surface of the tube to undergo plastic deformation.

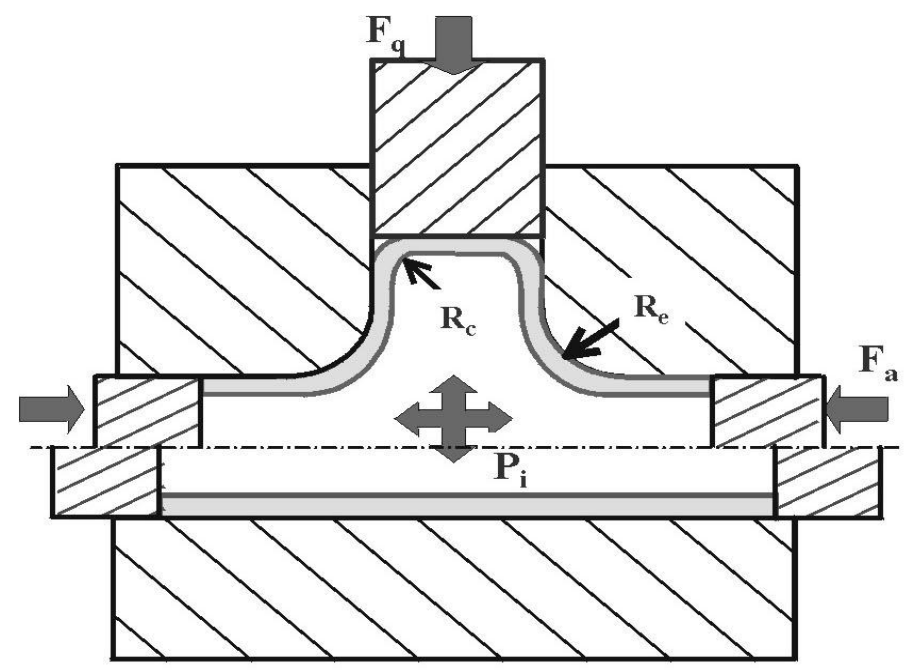

Fig.3 Elements of a typical thf process

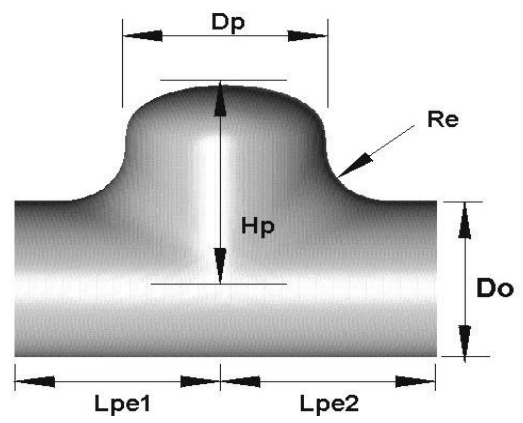

In Fig. 3 various elements of a typical THF process are shown as, (fa): axial force, (pi): internal pressure, (rc): corner radius, (re): fillet radius, (do): initial tube diameter, (dp): branch diameter or bulge width, (hp): branch or bulge height, (lp): distance between tube edge and branch.

\section{LITERATURE SURVEY}

Tube Hydroforming (THF) has been called with many other names depending on the time and country it was used and investigated. Bulge forming of tubes (BFTs) and liquid bulge forming (LBF) were two earlier terms, for instance. Hydraulic (or hydrostatic) pressure forming (HPF) was another form of name used for a while by some investigators. Internal high pressure forming (IHPF) has been mostly used within German manufacturers and researchers. In some periods, it was even called as "Unconventional Tee Forming". Throughout this paper, THF is used to describe the metal forming process whereby tubes are formed into complex shapes with a die cavity using internal pressure, which is usually obtained by various means such as hydraulic, viscous medium, elastomers, polyurethane, etc., and axial compressive forces simultaneously, Fig.1. Even though THF process has been in practical industrial use only more than a decade, development of the techniquesand establishment of the theoretical background goes back to 1940s. Manufacturing of seamless copper Fittings with T branches was investigated using internal pressure and axial load by Grey et al. [1]. Davis,[2] tested tubes of medium carbon steel under internal pressure and tensile axial load in order to determine their yield and fracture characteristics. Experimental and numerical studies were conducted to and the bursting pressure of thick-walled cylinders by Faupel, Crossland and Dietmann [3-5] during 1950s and 1960s. In 1960s, experimental and theoretical investigations on instability of thin walled cylinders were performed by many researchers at different countries [6-8]. Fundamental investigations on thin- and thick-walled cylinders helped theoretical improvements in LBF operations. Use of hydrostatic pressure in metal forming processes, in particular, for bulging of tubular parts was first reported by Fuchs [9]. In [9], the author reported experimental studies on expansion and bulgingof copper tubes using hydraulic pressure. Ogura and Ueda

$5^{\text {th }}$ National Conference RDME 2016, 10-1 $1^{\text {th }}$ March 2016.

48 | Page M.E.S. COLLEGE OF ENGINEERING, PUNE. 411001 
[10] presented their experimental resultson LBF of Tee shapes from low and medium carbon steel. Different configurations and number of Tee protrusions were formed using internal pressure and axial compressive loading. Proper forming zones were designed for Tee protrusions using experimental results. Experimental results for formingof "differential cases" were also disclosed in this paper. In the same period, Al-Qureshiet al. [11] performed bulging and piercing experiments of different materials including copper, steel and aluminium using polyurethane to provide internal pressure. They did not report use of axialloading in their experiments. In 1970s, research on different aspects of bulge forming continued both experimentally and theoretically by various authors. New shapes, materials, different tooling configurations and new machine concepts were introduced, whereas the fundamentals remained the same. For instance, instead of polyurethane, rubber and elastomers were used to provideinternal pressure [12]. He presented that greater circumferential expansion of thin-walled tubes was obtained using rubber forming methods than using hydraulic forming technique. Effect of friction between rubber and inner side of the tubes was also mentioned. Limb, et al. [13] performed BFTs of different materials with changing wall thickness. They reported that increasing the internal pressure gradually during the application of axial load gives the best results on thinning and thickening of tube wall at feeding zone due to thefriction between tube and die surface. In addition, experimentation of different lubricants such as PTFEfilm, colloidal graphite and Rocol RTD spray were carried out. In case of insufficient lubrication, low Tee protrusion heights were obtained as well as a bulged protrusion area resulted instead of a fully formed and ${ }^{-}$at area. With proper lubrication, it was reported that bulging of the Tee protrusion was obtained.

\section{PROBLEM OBJECTIVES AND PROCEDURE}

The present research work mainly incudes

- To study the loading pattern in the hydroforming process, i.eAxial feed, high pressure on the fluid, Clamping load on the die set and effective strain duringthe hydroforming.

- To study the relationship between maximum branch height and wall thickness.

- Comparison of the hydroforming simulation results from solid-works with the experimental results.

- To analyse thefactors influencingprocessfailures.

The following steps are followed to perform simulation in thesolid-works software.

1. Solid modelling of process setup

2. Apply Material condition to the model

3. Apply contacts

4. Apply boundary conditions\&Loading

5. Perform simulation

\section{ANALYTICAL ANALYSIS}

The fundamentals of tube hydroforming is the tube, which is first held in dies then close both top and bottom dies and seal both the tube ends. Thenfill the tube with a liquid solution of a water-soluble material. The tube is then pressed from both the ends axially at the same time apply pressure on the liquid until the tube take the shape of the die cavity. Release the high pressure and then reverse the process and take out the component from die.

During this process we will come across various failures(Fig.4) such as;

1. The risk of bursting: This is a result of too high internal pressure and is initiated by a local neck in the tube wall.

2. The Buckling: This is the result of high axial feed and less wall thickness due to the section modulus of cross section.

3. The Wrinkling: This is due to the long tube length this problem will get away during final high pressure forming. 


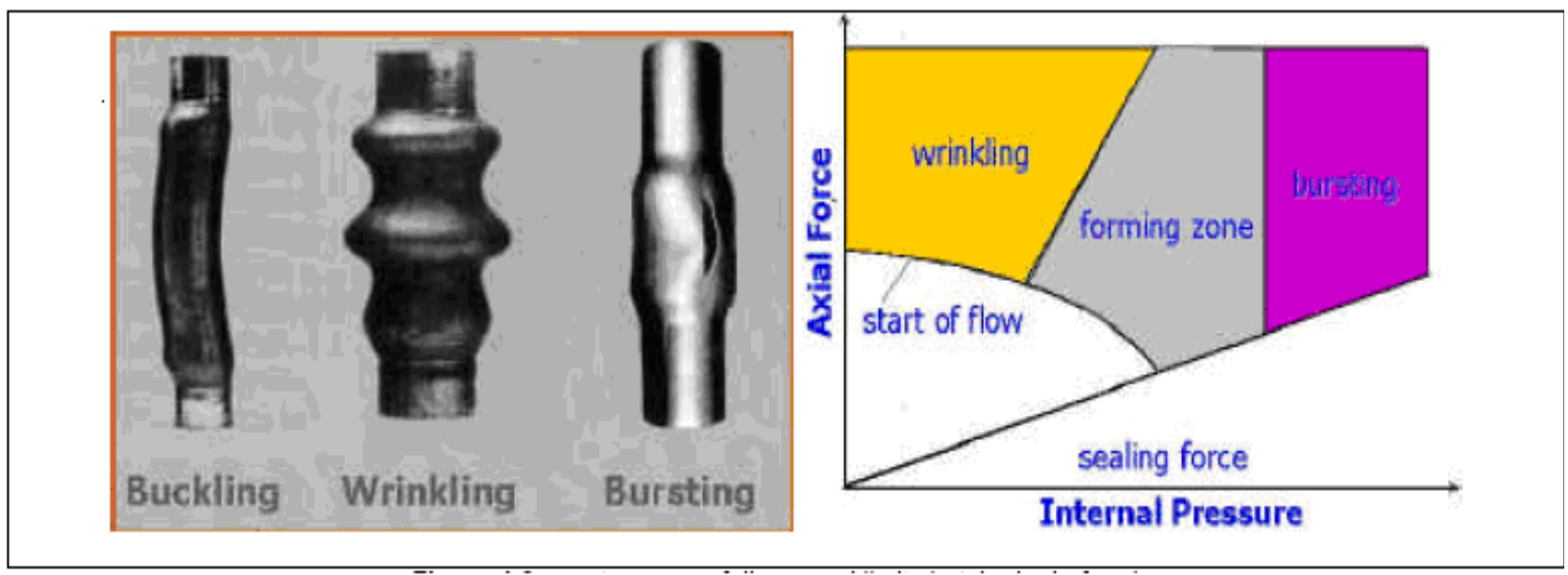

Fig. 4 Failures and limits in tube hydroforming

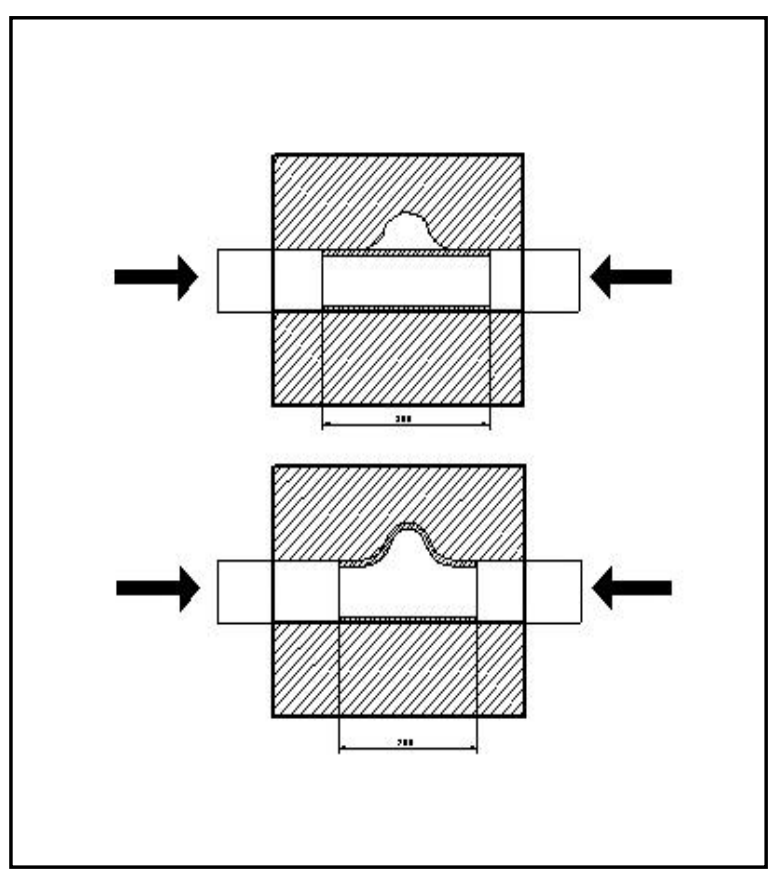

Fig.5Tube and die

.Table 1: Dimensions of the tube

\begin{tabular}{|c|c|c|c|}
\hline $\begin{array}{c}\text { LengthL } \\
(\mathrm{mm})\end{array}$ & $\begin{array}{c}\text { Internaldiameter } \\
\text { of tubeDi } \\
(\mathrm{mm})\end{array}$ & $\begin{array}{c}\text { Outerdiameter } \\
\text { of tubeDo }(\mathrm{mm})\end{array}$ & $\begin{array}{c}\text { Tube } \\
\text { Thicknesst }_{\mathrm{o}} \\
(\mathrm{mm})\end{array}$ \\
\hline 300 & 97.6 & 101.6 & 2 \\
\hline
\end{tabular}

Table 1 shows dimensions of the tube under consideration and Table 2 shows the mechanical characteristics AISI 304.

Table2: Mechanical characteristics AISI 304

\begin{tabular}{|c|c|}
\hline Material Name & AISI 304 \\
\hline Default Failure Criteria & Max Von Mises Stress \\
\hline Yield strength & $230 \mathrm{~N} / \mathrm{mm} 2$ \\
\hline
\end{tabular}

$5^{\text {th }}$ National Conference RDME 2016, 10-1 $1^{\text {th }}$ March 2016.

M.E.S. COLLEGE OF ENGINEERING, PUNE. 411001 


\begin{tabular}{|c|c|}
\hline Tensile Strength & $560 \mathrm{n} / \mathrm{mm} 2$ \\
\hline Elastic modulus & $190000 \mathrm{n} / \mathrm{mm} 2$ \\
\hline Poisson's ratio & 0.29 \\
\hline Mass Density & $8000 \mathrm{~g} / \mathrm{cc}$ \\
\hline Shear modulus & $75000 \mathrm{~N} / \mathrm{mm} 2$ \\
\hline Thermal Expansion Coefficient & $1.8 \mathrm{e}-005 /$ Kelvin \\
\hline
\end{tabular}

4.1 Theoretical Calculations

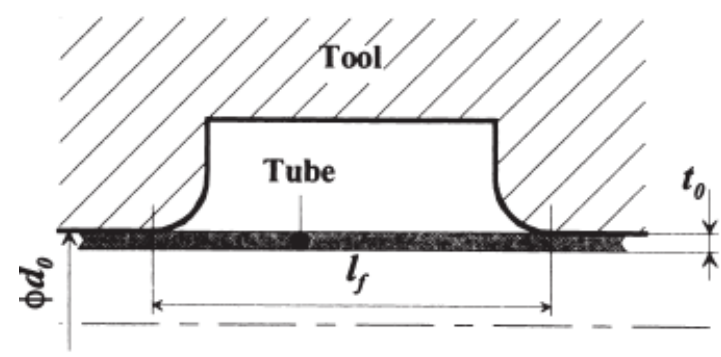

Fig. 6 Illustration of free tube length

4.2. Free tube length

$$
\begin{aligned}
& \text { If } 20 \leq \frac{D_{0}}{t_{0}} \leq 45 \rightarrow \text { If } \leq 2 \mathrm{D}_{0} \\
& \text { If } \frac{D_{0}}{t_{0}}>45 \rightarrow \text { If }<<2 \mathrm{D}_{0} \\
& \text { If } \frac{D_{0}}{t_{0}}<20 \rightarrow \text { If }>2 \mathrm{D}_{0}
\end{aligned}
$$

4.3 Corner radius of the die

$$
\mathrm{R}_{1}=3 \mathrm{t}
$$

4.4 Internal Pressure Limits

1. Internal pressure at yieldingby;

$$
\begin{aligned}
& \left(\mathrm{p}_{i}\right)_{y}=\sigma_{y} \frac{2 t_{0}}{\left(D_{0}-t_{0}\right)} \\
& \left(\mathrm{p}_{i}\right)_{y}=\frac{2300 * 2 * 2}{(101.6-2)} \\
& \left(\mathrm{p}_{i}\right)_{y}=92.36 \mathrm{MPa}
\end{aligned}
$$

2. Maximum internal pressure can be calculated by; 


$$
\begin{aligned}
\left(\mathrm{p}_{i}\right)_{b} & =\sigma_{u} \frac{4 t_{0}}{\left(D_{0}-t_{0}\right)} \\
\left(\mathrm{p}_{i}\right)_{b} & =\frac{5600 * 4 * 2}{(101.6-2)} \\
\left(\mathrm{p}_{i}\right)_{b} & =449.8 \mathrm{MPa}
\end{aligned}
$$

4.5 Sealing force

$$
\begin{aligned}
& F_{\text {sealing }}=\pi R_{0} t_{0} \sigma_{y} \\
& F_{\text {sealing }}=3.142 * 50.8 * 2 * 92.36 \\
& F_{\text {sealing }}=29,483 \mathrm{~N}
\end{aligned}
$$

4.6 Friction force

$$
\begin{aligned}
& F_{\text {friction }}=\mu p_{i} \pi d_{0}\left(l_{0}-s\right) \\
& F_{\text {friction }}=0.03 * 449.8 * 3.142 * 101.6 *[300-(3.142 * 101.6)] \\
& F_{\text {friction }}=82,824 \mathrm{~N}
\end{aligned}
$$

\section{MODELLING AND SIMULATION}

\subsection{Modelling of Tube, Dies and Axial Plungers}

The solid models of tubular blank, top die and bottom die, axialplungers with proper dimensions are shown below figures. Fig.7 shows solid model tubular blank.

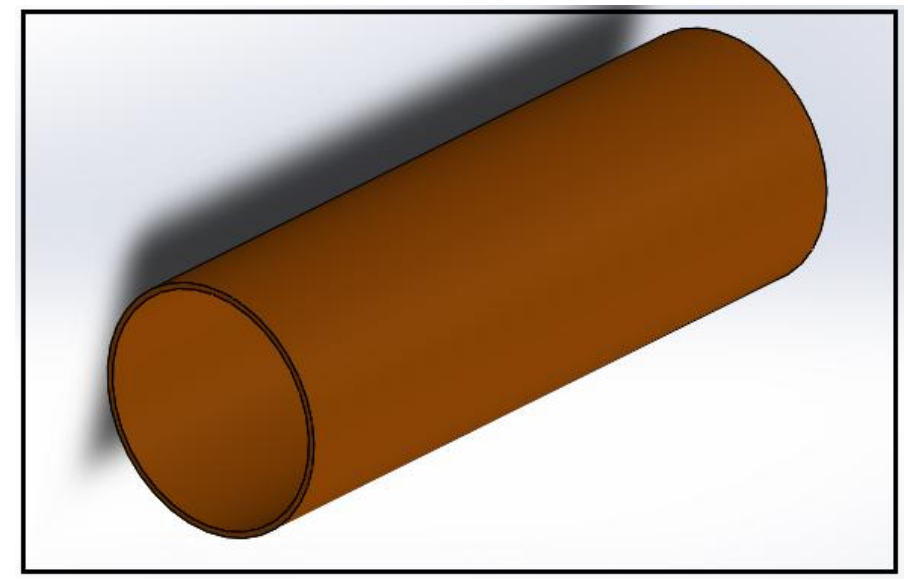

Fig. 7 Solid model of tubular blank

\subsubsection{Tube Blank Details}

Length $\mathrm{L}=300 \mathrm{~mm}$

Internal diameter $\mathrm{D}_{i}=97.6 \mathrm{~mm}$

Outer diameter of tube $\mathrm{D}_{0}=101.6 \mathrm{~mm}$

Thickness of tube $\mathrm{t}_{0}=2 \mathrm{~mm}$ 
5.1.2 Die, Plunger for Free Bulge

Two upper and lower dies are modelled by using Solid-works as shown in Fig.8. Model of the top die is, as shown in the Fig. 8 and Model of the bottomdie is, as shown in the Fig.9.

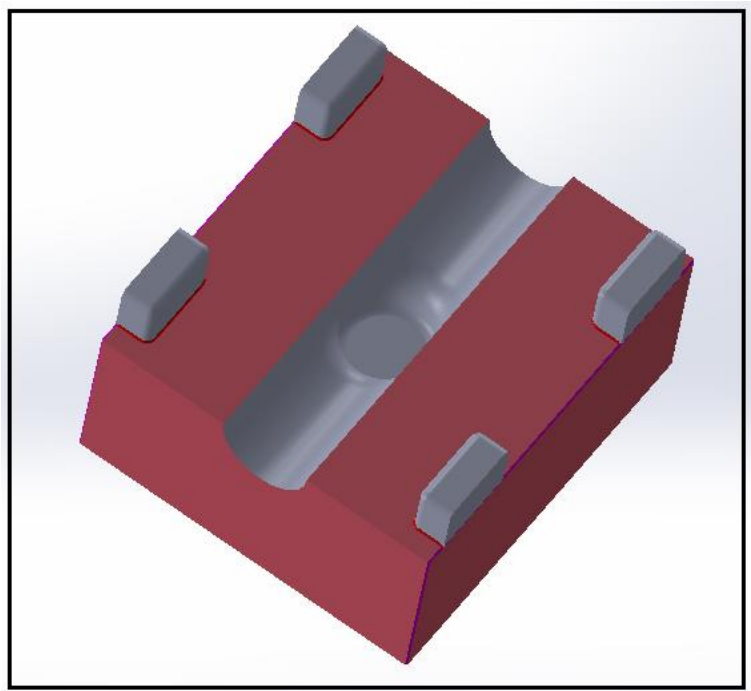

Fig. 8 Solid model of top die for free bulge forming

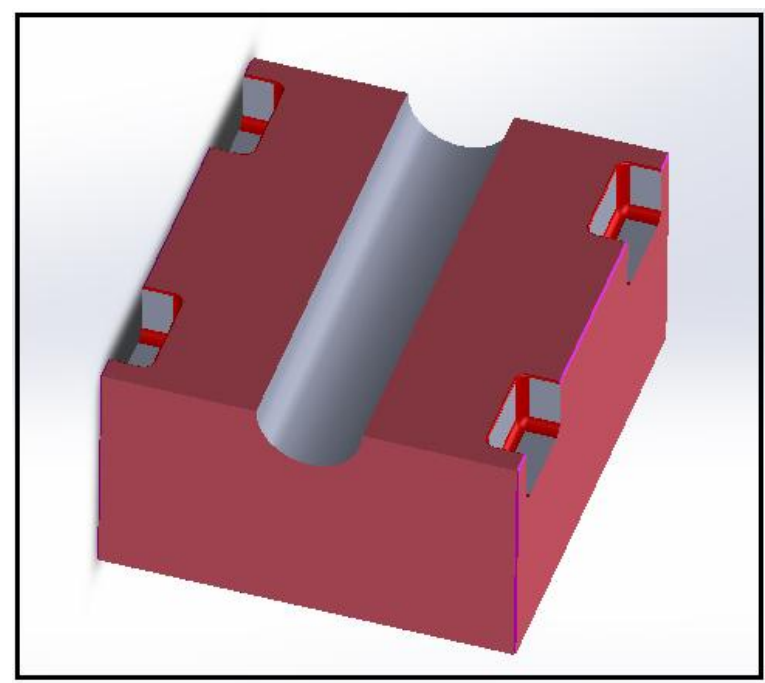

Fig.9 Solid Model of bottom die for free bulge forming

Die length $\mathrm{L}_{\mathrm{d}}=500 \mathrm{~mm}$ (Length perpendicular to tube axis)

Width $\mathrm{b}=400 \mathrm{~mm}$ (Width parallel to the tube axis)

Corner radius $\mathrm{R}_{1}=30 \mathrm{~mm}$ Tube cavity diameter

$D_{0}=101.6 \mathrm{~mm}$

Height of die $\mathrm{H}=200 \mathrm{~mm}$

Table.3 Branchheightcomparison ExperimentalandSimulationresults

\begin{tabular}{|c|c|}
\hline Parameter & Free-bulge \\
\hline MaximumInternalpressure(MPa) & 1000 \\
\hline MaximumFeed(mm) & 25.37 \\
\hline
\end{tabular}




\begin{tabular}{|c|c|}
\hline Branchheight $(\mathrm{mm})($ Experimental $)$ & 60 \\
\hline Branchheight $(\mathrm{mm})($ Simulation$)$ & 50.8 \\
\hline
\end{tabular}

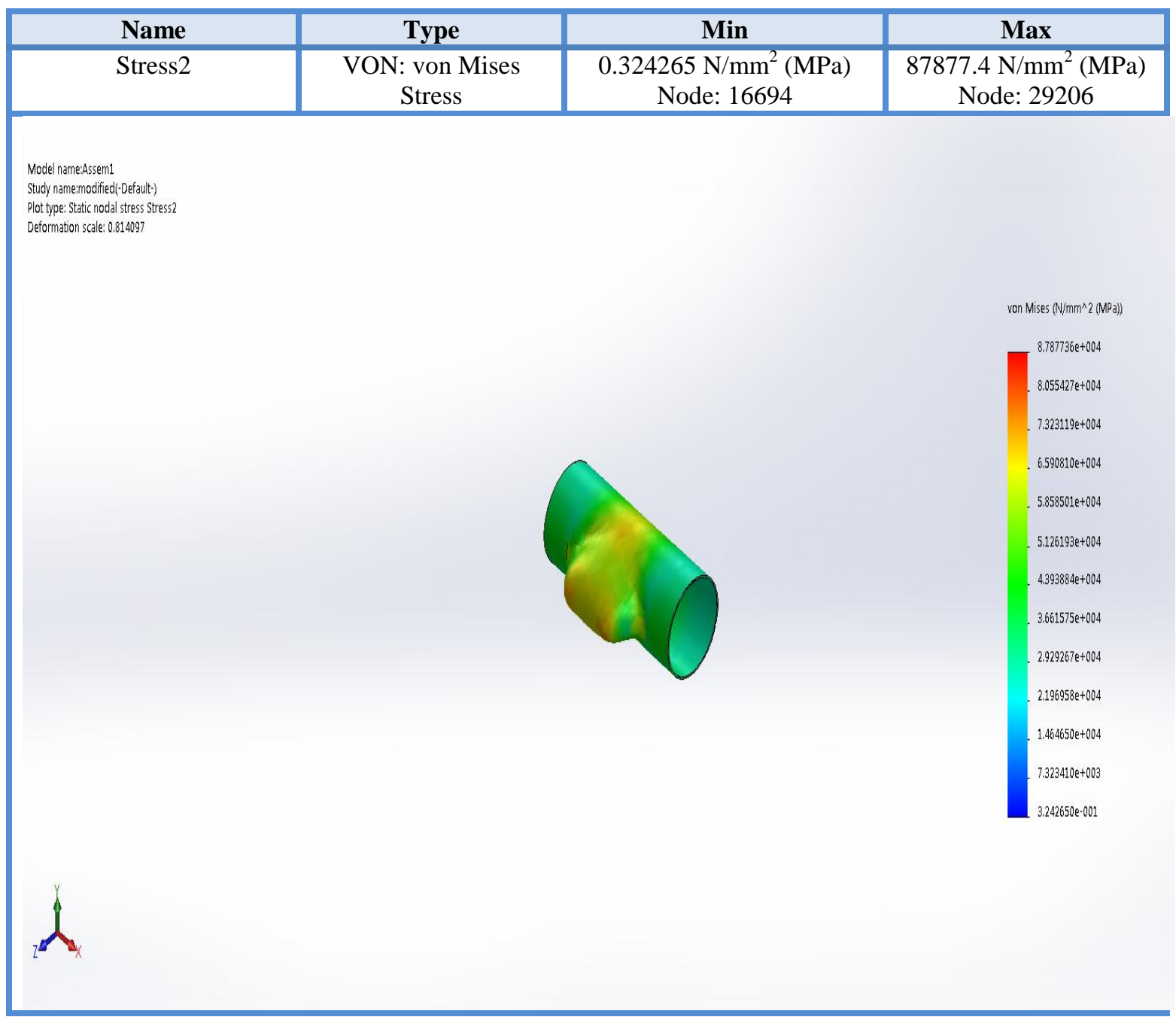

Fig. 10 FEA of Tube hydroforming

\section{RESULTS AND DISCUSSION}

Fig. 10 shows the finite element analysis of tube. From Fig.10 it is observed that the minimum value of the von Mises Stressis $0.324265 \mathrm{~N} / \mathrm{mm}^{2}$ (MPa) and maximum value of the von Mises Stress is $87877.4 \mathrm{~N} / \mathrm{mm}^{2}(\mathrm{MPa})$. After comparing these values of stresses with design stresses it is seen that the design is safe and the can be manufactured by Tube hydroforming. The influence of the axial movement and pressure applied on the confined fluid (water) in the tube is recorded and it is correlated as under- "The axial movement of tube is directly related with change in internal volume of the tube".

\section{CONCLUSION}

Hydroforming has come to be applied to manufacturing of automobile components over the last 50 years, but its problem was that forming conditions were complicated and difficult to understand. Through test forming of specimens of a simple shape by HF and FEM analysis of the test procedures, the influences of the loading path of internal pressure and axial feeding and the properties of material tubes over the forming process by HF were clarified. In addition, a compact design of the HF machine was developed. In the past these practices were very hefty and expensive. While these studies, the development have expanded for the application of HF, but still there 
some problems remainsunsolved, such as the technique for joining a work formed by HF with another component. Solution of these problems is essential forfurther expansion of the application of HF.

\section{References}

[1] J.E. Grey, A.P. Devereaux, W.N. Parker, Apparatus for making wrought metal T's, US Patent 2,203,868 June (1939).

[2] E.A. Davis, Yield and fracture of medium-carbon steel under combined stress, J. Appl. Mech. (1945) A13-A24.

[3] J.H. Faupel, Yield and bursting characteristics of heavy wall cylinders, Trans. ASME ( July 1956).

[4] B. Crossland, S.M. Jorgensen, J.A. Bones, The strength of thickwalled cylinders, ASME J. Eng. Ind. (May 1959).

[5] H. Dietmann, The - owbehavior of thick-walled cylinders under internal pressure, BanderBlecheRohre 8 (3) (1967) 143-149 (in German).

[6] P.B. Mellor, The ultimate tensile strength of thin-walled shells and circular diaphgrams subjected to hydrostatic pressure, Int. J. Mech. Sci. 1 (1960) 216-228.

[7] N.A.Weil, Tensile instability of thin-walled cylinders of ®nite length, Int. J. Mech. Sci. 5 (1963) 487-506.

[8] D.M. Woo, The analysis of axisymmetric forming of sheet metal and the hydrostatic bulging process, Int. J. Mech. Sci. 6 (1964) $303-317$.

[9] F.J. Fuchs, Hydrostatic pressure Đ its role in metal forming, Mech. Eng. (April 1966) 34-40.

[10] T. Ogura, T. Ueda, Liquid bulge forming, Metalworking Prod. (April 1968) 73-81.

[11] H.A. Al-Qureshi, P.B. Mellor, S. Garber, Application of polyurethane to the bulging and piercing of thin-walled tubes, in: Proceedings of the Ninth International MTDR Conference, Birmingham, UK, September 1968, pp. 319-338.

[12] H.A. Al-Qureshi, Comparison between the bulging of thin-walled tubes using rubber forming technique and hydraulic forming process, Sheet Metal Ind. (July 1970) 607-612.

[13] M.E. Limb, J. Chakrabarty, S. Garber, P.B. Mellor, The forming of axisymmetric and asymmetric components from tube, in: Proceedings of the 14th International MTDR Conference, 1973, pp. 799-805.

\section{Nomenclature:-}

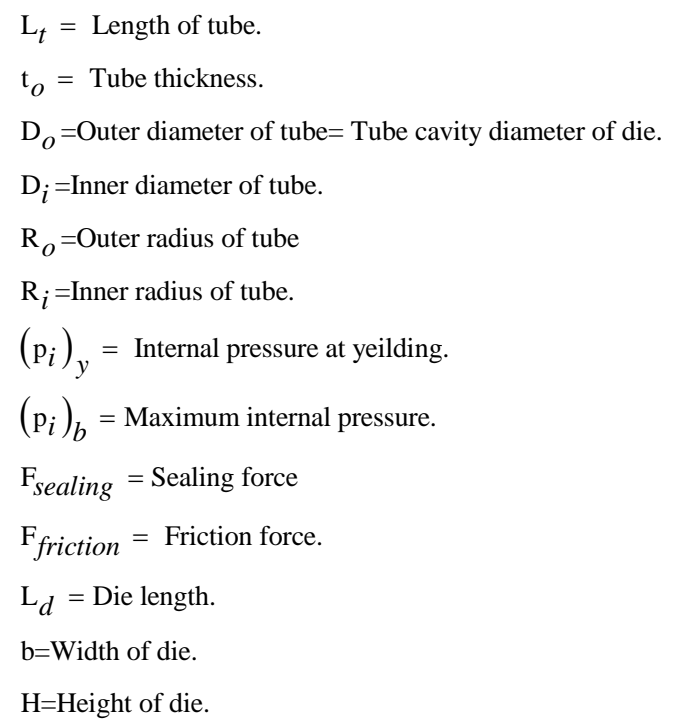

